\title{
ANÁLISE DO DESENVOLVIMENTO PSICOMOTOR EM INDIVÍDUOS COM DEFICIÊNCIA AUDITIVA
}

Deborah Cristina Gonçalves Luiz Fernani ${ }^{1,2}$, Janaina Andrade dos Santos ${ }^{1}$, Denise Cordeiro de Souza ${ }^{1}$, Vivian Andrade Sabo Ruy ${ }^{1}$, Francis Lopes Pacagnelli ${ }^{1}$, Carlos Bandeira de Mello Monteiro ${ }^{2,3}$, Maria Tereza Artero Prado ${ }^{1,2}$

${ }^{1}$ Universidade do Oeste Paulista - UNOESTE, Faculdade de Ciências da Saúde, Presidente Prudente - SP. ${ }^{2}$ Faculdade de Medicina do ABC, Laboratório de Escrita Científica, Santo André - SP. ${ }^{3}$ Universidade de São Paulo - USP, Escola de Artes, Ciências e Humanidades, São Paulo - SP. E-mail: deborah@unoeste.br.

\section{RESUMO}

O objetivo deste estudo foi analisar o desenvolvimento psicomotor de indivíduos com deficiência auditiva. A amostra foi composta por 12 indivíduos (média de idade de 9,16 2,69 anos): 6 apresentavam deficiência auditiva (DA) e 6 eram normoauditivos (NA) pareados por sexo e idade. Foi realizado exame audiológico que confirmou a ausência de déficit auditivo (NA) e classificou os indivíduos com DA. $\mathrm{Na}$ avaliação do desenvolvimento psicomotor utilizou-se a Bateria Psicomotora. Os dados foram apresentados de forma descritiva com média e desvio-padrão. As médias das pontuações em todos os fatores avaliados em ambos os grupos apresentaram valores acima ou igual a 3 , sendo classificados como perfil eupráxico. Os indivíduos apresentaram semelhança na pontuação final e obtiveram média de 23,83 na DA e 24,83 pontos na NA. Neste estudo ambos os grupos demonstraram desenvolvimento psicomotor adequado para a idade e não houve diferença com à presença de DA.

Palavras-chave: Desenvolvimento Infantil; Deficiências do Desenvolvimento; Transtornos de Audição; Perda Auditiva.

\section{ANALYSIS OF PSYCHOMOTOR DEVELOPMENT IN INDIVIDUALS WITH HEARING IMPAIRMENT}

\begin{abstract}
The aim of this study was to analyze the psychomotor development of individuals with hearing impairment. The sample was composed by 12 individuals (mean age of 9.16 \pm 2.69 years): 6 had hearing impairment $(\mathrm{HI})$ and 6 had normal hearing (NH) matched for sex and age. Audiological examination was realized which confirmed the absence of hearing deficit (NH) and classified individuals with HI. In the evaluation psychomotor development was used Bateria Psicomotora. The data were showed descriptively with mean and standard deviation. The mean scores in all factors evaluated in both groups showed values above or equal to 3, being classified as eupraxico profile. The individuals had a similar final score and obtained an mean of $23.83 \mathrm{in} \mathrm{HI}$ and 24.83 points in $\mathrm{NH}$. In this study both groups demonstrated appropriate psychomotor development for age and had no difference with the presence of HI.
\end{abstract}

Keywords: Child Development; Developmental Disabilities; Disorders of Hearing; Hearing Loss. 


\section{INTRODUÇÃO}

A deficiência auditiva (DA) é a diminuição ou perda da audição com redução da capacidade de percepção dos sons e dificuldade de compreensão das palavras, pode ser desenvolvida desde o nascimento ou adquirida devido a doenças, tais como: sequelas neurológicas devido a meningite, encefalite, entre outras ${ }^{1}$. Estas doenças podem acarretar vários problemas na aprendizagem o que contribui negativamente no desenvolvimento da linguagem, social e emocional ${ }^{2}$. Quando o diagnóstico é descoberto precocemente pode-se diminuir ou evitar estes acometimentos e quanto mais rápido este é detectado, menores são as dificuldades e maiores são as chances de favorecimento para o desenvolvimento global. Esse caráter preventivo na DA advém de políticas públicas focadas no diagnóstico e intervenção precoce ${ }^{3}$.

Segundo Northern e Downs ${ }^{4}$, a deficiência auditiva pode ser classificada de acordo com a localização da alteração, a qual pode ser dividida em perda auditiva condutiva, neurosensorial ou sensório neural e mista. A perda auditiva condutiva é caracterizada pela perda da condução aérea de sons. Já a perda auditiva neurosensorial, ocorre quando há presença de dano sofrido no órgão sensorial final ou nas células ciliadas localizadas dentro da cóclea, ou a disfunção pode ser um problema relacionado com os nervos auditivos nas vias superiores. Tradicionalmente, a lesão nas células ciliadas sensoriais não é facilmente diferenciada da lesão do nervo auditivo, de modo que a perda auditiva resultante é incluída na categoria neurosensorial. Já a perda auditiva mista, denomina-se a ocorrência concomitante das duas anteriormente citadas.

Estima-se que 42 milhões de pessoas acima de três anos de idade são portadores de DA moderada a profunda. Mais de $4 \%$ das crianças consideradas ao nascimento de alto risco são diagnosticadas como portadores de $D A^{5}$. Já no Brasil cerca de 9,7 milhões de pessoas possuem este deficiência, o que representa $5,1 \%$ da população brasileira. Deste total, cerca de dois milhões possuem a DA severa (1,7 milhões têm grande dificuldade para ouvir e 344,2 mil são surdos) e 7,5 milhões apresentam alguma dificuldade auditiva. No que se refere à idade, cerca de um milhão de crianças e jovens apresentam DA até 19 anos ${ }^{6}$.

A audição é considerada um órgão do sentido primordial para se obter a linguagem falada, além de influir nos processos psicológicos de integração e de experiências, como também pode comprometer a capacidade psicomotora ${ }^{7}$. Deste modo, além do acompanhamento que atenda todas as necessidades dos indivíduos com DA, é importante a avaliação do desenvolvimento 
psicomotor para verificar possíveis alterações, intervir caso necessário, minimizar problemas de aprendizagem e colaborar com o desenvolvimento global do indivíduo ${ }^{8}$. Sendo assim, este estudo teve como objetivo analisar o desenvolvimento psicomotor de indivíduos com deficiência auditiva.

\section{MÉTODOS}

Foram avaliados 12 indivíduos com média de idade de 9,16 $\pm 2,69$ anos, destes 6 apresentavam deficiência auditiva (DA) (3 do sexo feminino e 3 do masculino) e 6 eram normoauditivos (NA) pareados por sexo e idade. A amostra foi recrutada aleatoriamente em uma clínica privada de fonoaudiologia de Presidente Prudente/SP, com o auxílio da fonoaudióloga que realizou avaliação audiológica para verificar a presença ou não de DA. Os critérios de inclusão foram apresentar a audiometria com análise da presença da DA ou que comprovassem a normoaudição, além da autorização pelos responsáveis e sujeitos da pesquisa pelo termo de consentimento livre e esclarecido e o termo de assentimento. Foram excluídos indivíduos que apresentassem patologias do sistema nervoso central ou periférico, síndromes e/ou outras deficiências associadas. Este estudo foi aprovado pelo Comitê de Ética da
Universidade do Oeste Paulista (UNOESTE), sob o número CAAE: 19770013.8.0000.5515.

Para a avaliação audiológica foram classificadas as perdas auditivas de acordo com a localização, segundo Silman e Silverman $^{9}$. Na perda auditiva condutiva, os limiares de via óssea são menores ou igual a 15 dBNA e os limiares de via aérea são maiores do que 25 dBNA, com gap aéreoósseo maior ou igual a $15 \mathrm{~dB}$. E na perda auditiva neurosensorial ou sensório neural, os limiares de via óssea são maiores do que 15 dBNA e os de via aérea são maiores do que 25 dBNA, com gap aéreo-ósseo de até 10 dB. Já na perda auditiva mista, os limiares de via óssea são maiores do que 15 dBNA e os de via aérea são maiores do que 25 dBNA, com gap aéreo-ósseo maior ou igual a $15 \mathrm{~dB}$. Com relação ao grau da perda auditiva, a classificação foi baseada em Lloyd e Kaplan $(1978)^{10}$, para indivíduos maiores de 12 anos o grau de audição de $\leq 25$ dBNA significa audição normal, ou seja, com nenhuma dificuldade significativa; de 26-40 dBNA refere-se a perda auditiva de grau leve e dificuldade com fala fraca ou distante; 41-55 dBNA corresponde a perda auditiva de grau moderado com dificuldade com fala em nível de conversação; com 56-70 dBNA a fala deve ser forte e com dificuldade para conversação em grupo; $\geq 91$ dBNA trata-se de perda auditiva de grau profundo e pode não entender a fala amplificada, depende da 
leitura labial. Para indivíduos menores de 12 anos, Northern e Downs $(1984)^{11}$ classifica como $\leq 15$ dBNA como audição normal e com presença de sons da fala; de 16-25 dBNA o indivíduo apresenta perda auditiva discreta ou mínima, as vogais são ouvidas claramente e pode apresentar discreta dificuldade com as consoantes surdas; com 26-40 dBNA corresponde a perda auditiva de grau leve, ouve somente alguns dos sons da fala, os fonemas sonoros mais fortes; de 41-65 dBNA refere-se a perda auditiva de grau severo, o indivíduo não ouve os sons da fala de uma conversação normal; $\geq 96$ dBNA há perda auditiva de grau profundo, não ouve a fala ou outros sons.

Como instrumento de avaliação do desenvolvimento psicomotor, foi aplicada a Bateria Psicomotora (BPM) de Vitor da Fonseca $(1995)^{12}$, composta por sete fatores: tonicidade; equilíbração; lateralização; noção do corpo; estruturação espaço temporal; praxia global e praxia fina. Cada fator citado recebe a pontuação de um a quatro pontos, sendo: 1 ponto (apraxia) - ausência de resposta, realização imperfeita, incompleta, inadequada e descoordenada (muito fraco; disfunção evidentes e óbvias, objetiva-se dificuldades de aprendizagem significativas); 2 pontos (dispraxia) - realização fraca com dificuldade de controle e sinais desviantes (fraco, insatisfatório, disfunções ligeiras, objetiva-se dificuldades de aprendizagem); 3 pontos (eupraxia) - realização completa adequada e controlada (bom, disfunções indiscerníveis, não objetiva-se dificuldades de aprendizagem); 4 pontos (hiperpraxia) realização perfeita, precisa, econômica e com facilidade de controle (excelente, ótimo; objetiva-se facilidades de aprendizagem). Além disso, na BPM podem-se somar as pontuações de todos os fatores, obtendo-se a pontuação final para classificar o perfil psicomotor e associar a presença ou não de dificuldades de aprendizagem segundo a tabela a seguir.

Tabela 1. Escala de pontos da Bateria Psicomotora baseada em Fonseca ${ }^{12}$

\begin{tabular}{ccc}
\hline $\begin{array}{c}\text { Pontuação } \\
\text { Final }\end{array}$ & Tipo de perfil psicomotor & Dificuldades de aprendizagem \\
\hline $7-8$ & Deficitário & Significativas (moderadas ou severas) \\
$9-13$ & Dispráxico & Ligeiras (específicas) \\
$14-21$ & Normal & Ausentes \\
$22-26$ & Bom & Ausentes \\
$27-28$ & Superior & Ausentes \\
\hline
\end{tabular}

\section{RESULTADOS}

A análise dos dados apresentam-se de forma descritiva com média e desvio-padrão.
A classificação e os graus dos indivíduos com deficiência auditiva 
verificadas neste estudo estão descritas na Tabela 2. Pode-se observar que houve uma dispersão dos indivíduos de acordo com os comprometimentos auditivos.

Tabela 2. Caracterização da amostra de acordo com a classificação e grau da deficiência auditiva

\begin{tabular}{cl}
\hline Indivíduos & \multicolumn{1}{c}{ Classificação da deficiência auditiva } \\
\hline 1 & Moderada bilateral (Neurosensorial) \\
2 & Severa bilateral (Mista) \\
3 & OD Normal; OE Leve (Condutiva) \\
4 & OD Leve (Condutiva); OE Moderada \\
& (Condutiva) \\
5 & OD Normal; OE Moderada (Condutiva) \\
6 & Severa bilateral (Mista)
\end{tabular}

$\mathrm{OD}=$ Orelha direita, $\mathrm{OE}=$ Orelha esquerda.

As médias das pontuações em todos

os fatores avaliados pela Bateria Psicomotora em ambos os grupos apresentaram valores acima ou igual a 3 (Tabela 3), sendo classificados como perfil eupráxico: realização controlada e adequada da atividade.

$\mathrm{Na}$ pontuação final da bateria os indivíduos apresentaram pontuações semelhantes, sendo que os sujeitos com DA obtiveram média de 23,83 pontos e os NA de 24,83 pontos, classificados com perfil bom, com ausência de dificuldades de aprendizagem. Também não foi observada variação dos dados no que se refere à presença e grau de DA.

Tabela 3. Média e desvio-padrão da pontuação dos fatores avaliados pela Bateria Psicomotora

\begin{tabular}{ccc}
\hline & \multicolumn{2}{c}{ Indivíduos } \\
\cline { 2 - 3 } & DA & NA \\
\hline Tonicidade & $3,5 \pm 0,71$ & $3,17 \pm 0,41$ \\
Equilibração & $3,33 \pm 0,71$ & $3,67 \pm 0,52$ \\
Lateralização & $3,82 \pm 0,41$ & $3,83 \pm 0,41$ \\
Noção do corpo & $3,67 \pm 0$ & $3,5 \pm 0,55$ \\
Estruturação Espaço temporal & $3,5 \pm 0$ & $3,67 \pm 0,52$ \\
Praxia Global & $3,0 \pm 0$ & $3,0 \pm 0,63$ \\
Praxia Fina & $3,0 \pm 1,41$ & $3,33 \pm 0,82$ \\
Pontuação total & $23,83 \pm 3,54$ & $24,83 \pm 2,93$ \\
\hline
\end{tabular}

DA=deficiência auditiva, NA=normoauditivos. 


\section{DISCUSSÃO}

Os indivíduos avaliados neste estudo apresentaram as médias das pontuações em todos os fatores acima ou igual a 3 (perfil eupráxico). Além de obterem a classificação do perfil "bom" na pontuação final na análise do desenvolvimento motor. Estes dados corroboram com os de Silva et al. $(2008)^{13}$ que analisou o perfil psicomotor de 32 indivíduos com comprometimentos auditivos de 7 a 16 anos, com o mesmo instrumento utilizado nesta pesquisa, porém antes e após 72 aulas de LIBRAS (língua brasileira de sinais). Verificou que a maioria dos indivíduos apresentaram pontuação 3 nos fatores em ambas avaliações, somente o fator equilíbrio que mais de $70 \%$ da amostra apresentou pontuação 4 antes e após as aulas, o que demonstra o desenvolvimento psicomotor adequado destes indivíduos. Já o estudo de Costa et al. (2013) $)^{14}$ avaliou o desenvolvimento motor de 14 crianças surdas de 6 a 11 anos de uma escola do município de Cascavel/PR, no entanto para avaliação fez uso de outro instrumento denominado Escala de Desenvolvimento Motor, semelhante ao usado nesta pesquisa. Foi encontrado que $14,2 \%$ dos indivíduos apresentaram classificação de normal baixo, $21,3 \%$ inferior e $42,6 \%$ muito inferior, o que demonstra os déficits motores na maioria dos indivíduos avaliados, achados estes que divergem com os desta pesquisa.
Os indivíduos com DA e NA apresentaram semelhança na pontuação final da avaliação da bateria, ou seja, ambos não demonstraram déficit do desenvolvimento psicomotor. Este fato pode ser explicado por diversas atividades que os indivíduos com DA realizaram, tais como: intervenção precoce na área audiológica, inserção em escola regular, prática de várias atividades extras com ênfase na prática esportiva, o que pode ser indicador do bom desempenho durante a avaliação psicomotora. Vários autores ${ }^{15-18}$ descreveram sobre como a perda do desenvolvimento psicomotor na DA pode ser decorrente dos reflexos de privações ambientais, comportamento retraído e também pela hipercinesia, a qual pode gerar distúrbio de coordenação e alterar o desenvolvimento, o que não foi visualizado neste estudo, provavelmente devido aos indivíduos serem participativos em diversas atividades.

Pode citar como limitação deste estudo o número reduzido da amostra avaliada e sugere-se a análise do desenvolvimento psicomotor em individuos com DA em menor faixa etária para comprovação ou não da influência da deficiência em estágios precoces do desenvolvimento. 
CONCLUSÃO

Ambos os grupos apresentaram desenvolvimento psicomotor adequado para a idade, não sendo visualizada diferença no que se refere à presença de DA e ao seu grau neste estudo.

\section{REFERÊNCIAS}

1. Anjos LP, Queirós F, Pereira MC, Brandão $M$, Melo $A$, Lucena R. Prognóstico audiológico tardio relacionado à meningite em lactentes. Arq Neuropsiquiatr. 2004;62(34):635-40.

2. Dantas MBS, Anjos CAL, Camboim ED, Pimentel MCR. Resultados de um programa de triagem auditiva neonatal em Maceió. Rev Bras Otorrinolaring. 2009;75(1):58-63. DOI: http://dx.doi.org/10.1590/S0034-

\section{$\underline{72992009000100009}$}

3. Nogueira JCR, Mendonça MC. Assesment of hearing in a municipal public school students population. Braz.J.Otorhinolaryngol. 2011:77(6):71620.DOI: $\quad$ http://dx.doi.org/10.1590/S180886942011000600007

4. Northern JL, Downs MP. Audição na infância. $\quad 5^{\circ}$ edição. Williams Wilkins: Guanabara Koogan; 2005.

5. Silva LPA, Queiros F, LIMA I. Fatores etiológicos da deficiência auditiva em crianças e adolescentes de um centro de referência APADA em salvador BA. Rev Bras Otorrinolaring. 2006;72(1) 33-36. DOI: http://dx.doi.org/10.1590/S0034-

\section{6}

6. IBGE: Instituto Brasileiro de Geografia e Estatística. Censo Demográfico, Características gerais da população, religião e pessoas com deficiência. Acesso: 20 de junho 2013 Disponível em: http://www.ibge.gov.br/home/estatistica/po pulacao/censo2010/caracteristicas religiao

deficiencia/default caracteristicas religiao d eficiencia.shtm.2010.

7. Araújo SM, Meira JCM, Cantarelli EM. Equilíbrio estático em crianças portadoras de deficiência auditiva neurossensorial. Cad Uni ABC de Educação Física. 2001;(16): 56-70.

8. Castro GM, Viana TV, Alencar ML. Movimentos silentes: a educação psicomotora no tratamento de problemas de aprendizagem de alunos surdo. Diálogo Educação. 2007;7(20):81-89.

9. Silman $S$, Silverman CA. Basic audiologic testing. In: Silman S, Silverman CA. Auditory diagnosis: principles and applications. San Diego: Singular Publishing Group; 1997;44-52.

10. Lloyd LL, Kaplan H. Audiometric interpretation: a manual o basic audiometry. University Park Press: Baltimore, 1978;16-7.

11. Northern JL, Downs MP. Hearing in Children, $3^{\circ}$ ed. Williams, Wilins: Baltimore, 1984;89.

12. Fonseca V. Manual de observação psicomotora: significação psiconeurológica dos fatores psicomotores. Porto Alegre. Casa publicadora. 1995.

13. Silva FC, Aquino FJM, Bello SAF. Avaliação Psicomotora em crianças com comprometimentos auditivos submetidas ao ensino de libras. Anuário da Produção de Iniciação Científica Discente. 2008;11(12):139-46.

14. Costa AG, Dorst DB. Avaliação do desenvolvimento motor da criança surda no município de Cascavel. Revista Digital EFDeportes.2013 set; 18(184). Disponível em:<http://www.efdeportes.com/efd184/de senvolvimento-motor-da-crianca-surda.htm>. Acesso em: 5 Dez 2013. 
15. Moret ALM, Bevilacqua MC, Costa OA. Implante coclear: audição e linguagem em crianças deficientes auditivas pré-linguais. Pró-Fono Revista de Atualização Científica. 2007;19(3):295-304.

DOI:

http://dx.doi.org/10.1590/S0104-

$\underline{56872007000300008}$

16. Souza NA, Gorla, Jl, Araújo PF, Lifante $\mathrm{SM}$, Campana MB. Análise da coordenação motora de pessoas surdas. Arq. Ciênc. Saúde Unipar. 2008 set./dez; 12(3):205-11.

17. Angelo TSC, Bevilacqua MC, Moret ALM. Percepção da fala em deficientes auditivos pré-linguais usuários de implante coclear. Pró-Fono Revista de Atualização Científica. 2010;22(3):275-80. DOI: http://dx.doi.org/10.1590/S0104-

\section{0}

18. Gândara JP, Befi-Lopes DM. Tendências da aquisição lexical em crianças em desenvolvimento normal e crianças com alterações específicas do desenvolvimento da linguaguem. Rev. Da Sociedade Bras. De Fonoaudiologia. 2010;15(2):297-304. DOI: http://dx.doi.org/10.1590/S1516-

80342010000200024

Recebido para publicação em 31/07/2014

Revisado em 15/08/2014

Aceito em 20/08/2014 